\title{
Prensa en internet: nuevos modelos de negocio en el escenario de la convergencia
}

\author{
Por Andreu Casero-Ripollés
}

\begin{abstract}
Resumen: Internet está transformando el modelo de negocio de la prensa. El acceso gratuito a las noticias online, el aumento de la competencia y la crisis del sector han reabierto el debate sobre el futuro de los diarios en la era de la convergencia. El objetivo de este artículo es examinar las bases de los nuevos modelos de negocio de la prensa en la Red. El análisis se ocupa de las fuentes de ingresos de la información online: la publicidad y la venta del producto. La metodología elegida es el estudio de casos que se aplica a las estrategias empresariales de cuatro diarios digitales españoles: las versiones electrónicas de los dos principales periódicos de información general (Elpaís.com y Elmundo.es) y dos cibermedios exclusivamente digitales (Soitu y Factual).
\end{abstract}

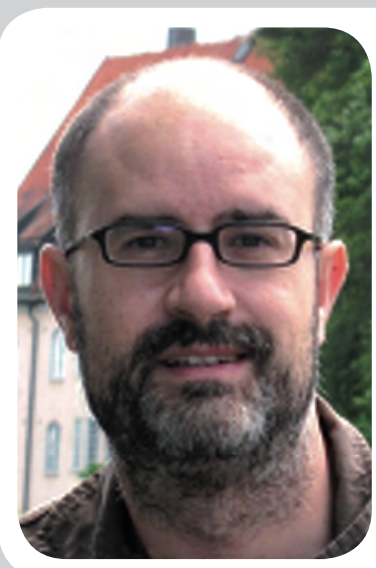
Andreu Casero-Ripollés es profesor titular del Departamento de Ciencias de la Comunicación de la Universitat Jaume I de Castelló, donde, además, es director del Grado en Periodismo. Es licenciado por la Universitat Autònoma de Barcelona y doctor por la Universitat Pompeu Fabra. Ha sido profesor invitado en la Università di Milano-Bicocca (Italia) y en la Universitè Paris 8 (Francia). Es autor del libro "La construcción mediática de las crisis políticas" y co-editor de "El desarrollo de la televisión digital en España". Recientemente, ha sido galardonado con el 5 Premio de Investigación Universitaria otorgado por la Asociación Catalana de Prensa Comarcal.

Palabras clave: Prensa, Internet, Modelo de negocio, Convergencia, España.

\section{Title: Newspapers on the internet: new business models in the convergence era}

Abstract: The internet is transforming the business model of the press. Free access to online news, increased competition and the crisis of the media sector has reopened the debate on the future of newspapers in the convergence era. The object of this paper it is to examine the basis of the new business models of the press on the Net. The analysis addresses the revenue sources for online information: advertising and sale of the product. The methodology is the case study, applied to the business strategies of four Spanish digital newspapers: the online versions of two main newspapers (Elpaís.com and Elmundo. es) and two pure digital players (Soitu and Factual).

Keywords: Newspapers, Internet, Business Model, Convergence, Spain.

Casero-Ripollés, Andreu. "Prensa en internet: nuevos modelos de negocio en el escenario de la convergencia". El profesional de la información, 2010, noviembre-diciembre, v. 19, n. 6, pp. 595-601.

DOI: 10.3145/epi.2010.nov05

\section{Introducción}

Internet supone un reto de grandes magnitudes para la prensa no sólo por los cambios que introduce en la producción y la distribución de la información, sino también en términos industriales. Plantea un desafío de primer orden para el modelo de negocio vigente en este sector, que se ve seriamente cuestionado en el contexto digital.

Dos son las causas que motivan esta problemática: el predominio de la gratuidad en el acceso a la información online, y la incapacidad de las empresas periodísticas para instaurar una fórmula viable para obtener recursos económicos de su actividad en internet. Esta situación llega, incluso, a provocar una paradoja: el consumo de noticias online aumenta de forma notable, pero los editores de prensa no consiguen traducir ese incremento de la demanda en ingresos financieros que hagan rentable su negocio. Es decir, no logran monetizar la ampliación de la audiencia.

Otro factor clave en esta ecuación es la asunción tardía por parte de los diarios del cambio del modelo informativo que entraña la convergencia digital. Los editores han desarrollado proyectos periodísticos online más como una reacción a los movimientos de sus competidores que como una búsqueda de nuevos horizontes de negocio, revelando su carácter conservador y defensivo (Boczkowski, 2006).

Además, la prensa asiste a un fuerte aumento de la competencia en la Red. El mercado de la información online se ve poblado por nuevos medios, como los confidenciales, los buscadores, los diarios exclusivamente digitales -pure players- o los derivados del periodis- 
mo ciudadano como los blogs o las redes sociales. El número de proveedores de noticias de actualidad se multiplica, generando innumerables ventanas donde informarse.

A este cuadro cabe añadir la profunda crisis por la que atraviesa la prensa (Larrañaga, 2009; Mateo; Bergés; Garnatxe, 2010). La gravedad de la misma es tal que en un año, de 2007 a 2008, los diarios españoles sufrieron un descenso de sus beneficios netos del $95 \%$, pasando de 232,9 millones a 11,9 millones de euros $(A E D E, 2009)$. En Estados Unidos los periódicos han perdido un $41 \%$ de sus ingresos entre 2007 y 2009 (PEJ, 2010). Este escenario es fruto de la suma de dos crisis: la estructural que la industria de la prensa venía padeciendo desde mediados de la década de los noventa, por un lado, y la recesión financiera que afecta a todas las economías mundiales desde 2007, por otro. El efecto más visible de la primera es el lento, pero progresivo, declive en las ventas de diarios (SánchezTabernero, 2008). En los últimos tres años, 20 países de los 30 integrantes de la Organización para la Cooperación y el Desarrollo Económico (OCDE) han registrado caídas en la difusión, siendo los más afectados Estados Unidos y Reino Unido, con bajadas del 30\% y del $21 \%$ respectivamente $(O C D E, 2010)$. España pierde, en ese mismo período, un $16 \%$.

Las razones de estos descensos son diversas pero las principales son la pérdida de influencia de los medios impresos frente a otros como la televisión o internet (López; Pereira, 2008), el alejamiento del público joven y la creciente orientación de los lectores hacia el consumo online de las noticias, que se ha incrementado del $18 \%$ al $31 \%$ de la población europea en los últimos cinco años según Eurostat. Sólo los países en desarrollo o las nuevas potencias internacionales, como China, India o Brasil, protagonizan incrementos en la venta de rotativos de pago (Dornelles, 2009).

La recesión financiera ha actuado como elemento acelerador que ha agudizado la crisis estructural de la prensa. El estancamiento económico ha afectado a uno de los pilares del sector: la publicidad. Ésta se ha reducido drásticamente en los últimos años. De 2007 a 2009 en Estados Unidos la publicidad en los diarios ha bajado un $43 \%$. En España, hasta el primer semestre de 2010, la disminución ha sido del 40,4\% (Infoadex, 2010). Unos datos que han disparado las alarmas, puesto que una media del $57 \%$ de los ingresos de los periódicos en los países de la $O C D E$ proceden de los anuncios $(O C D E, 2010)$. Una cifra que, en la prensa española de pago, supone el 43,4\% del total (AEDE, 2009).

En este contexto, el debate sobre el futuro de los diarios se ha reavivado. Especialmente, aquél que sitúa su foco de interés en la posible sustitución de la prensa en papel por la digital, bien sea por la extinción de la primera (Meyer, 2009), bien sea por su sustitución por dispositivos móviles, como el lector de libros electrónicos (Díaz-Noci, 2009). La refundación de los modelos de negocio de la prensa para adaptarlos a la convergencia digital se ha convertido en una necesidad imperiosa.

\section{"La fuerte crisis que vive el sector revela la necesidad de refundar el modelo de negocio de la prensa en la Red"}

\section{Objetivos, metodología y marco teórico}

Este artículo examina cuáles son las bases de los nuevos modelos de negocio de la prensa en internet. Para ello se centra en el estudio de las fuentes de financiación de la información online: la publicidad y la venta del producto en la Red. Aunque constituye un elemento importante, el estudio de los contenidos no se aborda aquí por motivos de espacio.

El marco teórico aplicado es el de la economía política de la comunicación (Mosco, 2009). Se adopta así una orientación macroeconómica para estudiar cómo es y cómo funciona el sistema mediático, desde una perspectiva estructural. Bajo este enfoque, la acción de los actores comunicativos viene condicionada por factores tecnológicos, económicos y políticos, y no únicamente por una de estas dimensiones en exclusiva.

Esta perspectiva se engloba dentro del contexto de la convergencia digital -integración de prensa escrita, radio-televisión e internet-. Ésta se configura como un proceso en desarrollo (Deuze, 2009) que supone la irrupción de nuevas lógicas que ponen de manifiesto la tensión entre el cambio y la tradición que define actualmente al periodismo en todas sus dimensiones (Mitchelstein; Boczkowski, 2009). No sólo posee un componente técnico sino que se extiende hacía el plano cultural (Jenkins, 2008). Afecta a los ámbitos tecnológico, empresarial, profesional y editorial del sistema mediático (García-Avilés, 2009). Por lo tanto tiene que ver directamente con los aspectos estructurales y organizativos, circunstancia que sitúa la reconfiguración de los modelos de negocio como un elemento esencial de estudio (Palacios; Díaz-Noci, 2009).

La convergencia propicia que las empresas comunicativas tiendan a incrementar su presencia en todos los mercados (Lawson-Borders, 2006). Para ello se extienden a través de la cadena de valor -tanto vertical como horizontalmente-, buscan sinergias y diversifican 
los soportes (Boczkowski, 2006) aplicando una distribución multiplataforma. Estos procesos no sólo favorecen la formación de grandes grupos de comunicación que aspiran a una creciente integración (Salaverría; García-Avilés, 2008) sino que sitúan a la reducción de costes como uno de los principales objetivos de la industria mediática (Mateo; Bergés; Garnatxe, 2010). En consecuencia, la convergencia supone una alteración de los escenarios vigentes.

La metodología puesta en práctica en este artículo se basa en los estudios de casos (Albarran, 2010; Salaverría; Negredo, 2008). Se exploran las estrategias desplegadas en internet por cuatro diarios españoles. Se trata, por un lado, de las versiones digitales de los dos principales periódicos de información general -Elpaís. com y Elmundo.es- y, por otro, de dos experiencias innovadoras implementadas por pure players o diarios con presencia exclusiva en la Red -Soitu y Factual. Estos ejemplos detallarán cómo la prensa está incorporando los nuevos modelos de negocio en la Red.

\section{Fuentes de financiación de la prensa en internet}

Tradicionalmente la prensa ha basado su obtención de ingresos en una doble financiación que implica tanto la venta del producto a los lectores como el recurso a la publicidad (Sonnac, 2009). Internet ha alterado tanto las prácticas de los consumidores como las de los anunciantes, provocando cambios en estas dos fuentes.

A nivel global la publicidad en internet ha crecido entre 2003 y 2008 un 32,4\% (PriceWaterhouseCoopers, 2009b), protagonizando el mayor aumento del sistema comunicativo y acaparando un $10 \%$ del total del mercado publicitario internacional. En España el incremento de los anuncios online es también un hecho. Su despegue se produce en 2006 -gráfico 1- y ha provocado que, actualmente, la Red sea el tercer medio por inversión publicitaria, tras la televisión y los diarios impresos, con un 11,6\% del total (Infoadex, 2010). Estos datos revelan una sostenida migración de la publicidad hacia el entorno digital, pero también ponen de manifiesto que esta transición se está llevando a cabo lentamente.

Específicamente en lo que se refiere a la prensa, la inversión publicitaria online creció un 17,2\% a nivel global en 2008 (PriceWaterhouseCoopers, 2009a). No obstante, en los países avanzados, esta fuente de financiación todavía resulta escasa puesto que en 2009 suponía alrededor del $4 \%$ del total de ingresos de un diario (OCDE, 2010).

Las previsiones de futuro indican que, mientras la publicidad en los diarios impresos descenderá a un ritmo de un $4,5 \%$ anual hasta 2013 , el volumen de anuncios captado por la prensa online crecerá un $6,8 \%$ anual en el mismo período (PriceWaterhouseCoopers, 2009a). Sin embargo, todo señala que la publicidad en exclusiva resultará insuficiente para garantizar los ingresos necesarios a la industria de la prensa (Sonnac, 2009). Varios datos avalan esta afirmación. Por un lado, a pesar de su crecimiento, la publicidad online representará únicamente un $9,1 \%$ del total del mercado de los periódicos en 2013. Por otro, pese a los aumentos en internet, la publicidad en la industria de los diarios perderá, en conjunto, un $3,7 \%$ de su volumen anualmente hasta 2013 (PriceWaterhouseCoopers, 2009a).

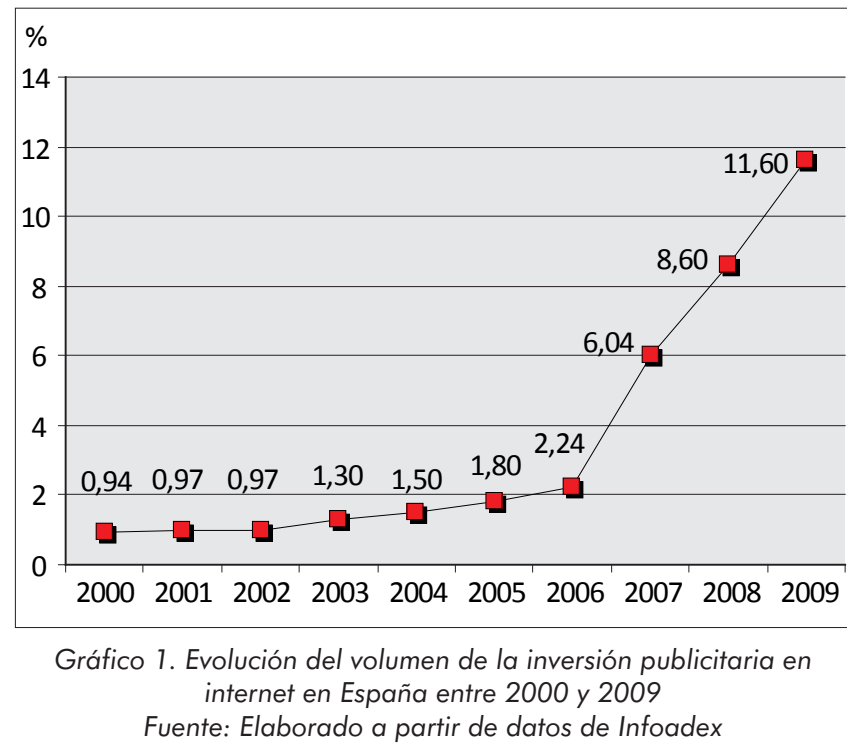

La fuente de financiación que ha sufrido un mayor cambio en internet, en el caso de la prensa, es la venta del producto. Los lectores pueden acceder a grandes cantidades de información de forma gratuita e, incluso en algunos casos, pueden consumir libremente online los mismos contenidos que ofrece la edición impresa del periódico de pago. Una circunstancia que provoca desajustes en la economía de los diarios, llegando al extremo de generar una canibalización entre las dos versiones (Rojo-Villada, 2008).

En la Red se ha consolidado una cultura de la gratuidad, en la que el acceso a la información de actualidad se realiza sin efectuar ningún pago y, además, se genera una resistencia cada vez mayor a éste entre los usuarios (Miguel-de-Bustos; Casado-del-Río, 2010). Las personas dispuestas a pagar por consumir noticias online se sitúan entre el $10 \%$ y el $20 \%$ del total de lectores, según diferentes estudios (WAN, 2010; PEJ, 2001; PriceWaterhouse Coopers, 2009b). El arraigo de la gratuidad es tal que el $74 \%$ de los usuarios buscaría otro medio de acceso libre si su sitio web favorito decidiera cobrar por las noticias (WAN, 2010). Esta tendencia se ha visto reforzada por la web 2.0, cuya lógica se basa en la colaboración y la cooperación. 
La gratuidad supone un cambio en el modelo de negocio de los diarios, ya que su estrategia debe orientarse a generar grandes audiencias y a monetizarlas después mediante los anuncios (Sonnac, 2009). Algunos defienden que el libre acceso a la información aumentará el consumo de noticias, atrayendo, así, a la financiación publicitaria (Anderson, 2009). La publicidad se convierte en el único recurso de la economía de la prensa, como en el caso de la televisión en abierto y la radio. Eso puede condicionar los contenidos informativos y hacer que éstos deriven hacia una excesiva homogeneización, hacia el predominio de temas triviales y sensacionalistas que busquen el impacto y el entretenimiento, hacia la pérdida de la información local y, en suma, hacia un descenso de la calidad del producto periodístico (OCDE, 2010).

El reducido volumen de los ingresos procedentes de la publicidad online está provocando que numerosos editores de prensa barajen la posibilidad de implantar sistemas de pago para el acceso a sus noticias en la Red. El éxito de estas fórmulas sólo es posible si la información ofrecida posee un fuerte valor añadido. Cuatro son los puntos clave en este sentido:

- brindar un contenido relevante para las vidas de los usuarios, basado en la personalización y la especialización (Rodríguez-Martínez; Codina; PedrazaJiménez, 2010; Martínez-Molina, 2010; Micó-Sanz; González-Molina, 2010),

- potenciar la documentación para mejorar el producto periodístico y ofrecer nueva información (Marcos-Recio; Edo; Flores; García-Alonso; Parra, 2008),

- que la información sea única y exclusiva -no accesible en otros sitios web- (Miguel-de-Bustos; Casado-del-Río, 2010) y, finalmente,

- que las empresas periodísticas acuerden una migración en bloque hacia métodos de pago (Rojo-Villada, 2008).

A partir de la dicotomía pago-gratuidad, internet permite la configuración de diversos modelos de negocio para la prensa:

- gratuidad (advertiser-supported): es la fórmula más extendida y convierte a la publicidad en el único recurso de financiación. Algunos diarios que aplican este método son Elpaís.com o Lainformación.com, en España, y The guardian, en el contexto europeo.

- pago total (pay-wall): se basa en el cobro por el consumo de información y admite fórmulas como la suscripción -anual o mensual- o el pago por uso -payper-use-, que engloba el pago por día/semana o por artículo leído, siguiendo el modelo de micropagos de iTunes. El principal defensor de este modelo es Rupert
Murdoch, propietario del grupo News corporation, quien lo ha adoptado en sus diarios online Wall Street journal, con una suscripción anual de 79 dólares, y The times, que ha perdido el $77 \%$ de sus visitas -equivalentes a 1,2 millones de usuarios- tres meses después de la implantación del pago.

- metered model: permite el acceso gratuito a un número limitado de artículos, diez en el caso del $\mathrm{Fi}$ nancial times -que cuenta con 250.000 abonados en 2010-, y, una vez superada esa cifra, los lectores deben pagar. Se pretende que la gratuidad actúe como un imán. En enero de 2011, se sumará a esta fórmula The New York times.

- freemium: combina el consumo en abierto a las noticias de última hora con el cobro por el acceso a informaciones dotadas de alto valor añadido. Junto al diario español Elmundo.es, también ha apostado por esta fórmula el grupo Independent news and media, poniéndolo en marcha en 13 periódicos regionales de Irlanda.

- donaciones: es un método poco aplicado que sitúa a la prensa fuera del mercado y basa sus ingresos en contribuciones voluntarias. Propublica.org, en Estados Unidos, y Periodismohumano.com, en España, constituyen dos ejemplos.

La exploración de nuevos caminos pasa también por la aplicación de estrategias en la gestión de los contenidos adaptadas a la convergencia desde una óptica empresarial. En este sentido sobresalen la búsqueda de sinergias entre los diferentes medios de un mismo grupo de comunicación, la integración de redacciones (Salaverría; Negredo, 2008) y, especialmente, la potenciación de la distribución multiplataforma (Albarran, 2010). Ésta consiste en hacer circular el mismo contenido por diferentes canales y que, por una u otra vía, llegue al público, captando su atención (Jenkins, 2008; Micó-Sanz; Masip; Barbosa, 2009).

\section{"El consumo de noticias online aumenta de forma notable pero eso no se traduce en ingresos"}

\section{Estudio de casos}

Las estrategias desplegadas en internet por los dos principales diarios españoles de información general han seguido caminos diferentes. Elpaís.com fue pionero en Europa en la introducción del modelo de negocio basado en el pago total. En noviembre de 2002 pasó a cobrar por el acceso a los contenidos de su edición onli- 
ne, exceptuando la portada y los artículos de opinión. La adopción de esta fórmula afectó negativamente a sus ingresos. Por un lado, no logró captar la atención del público, puesto que tras dos años de funcionamiento de este sistema el número de subscriptores era sólo de 40.500 personas (Salaverría, 2007). Este descenso de la audiencia condujo a un importante retroceso de la publicidad. En 2005 Prisacom, la empresa editora de Elpaís.com, redujo un $30 \%$ sus ingresos por anuncios. En consecuencia, el impacto negati-

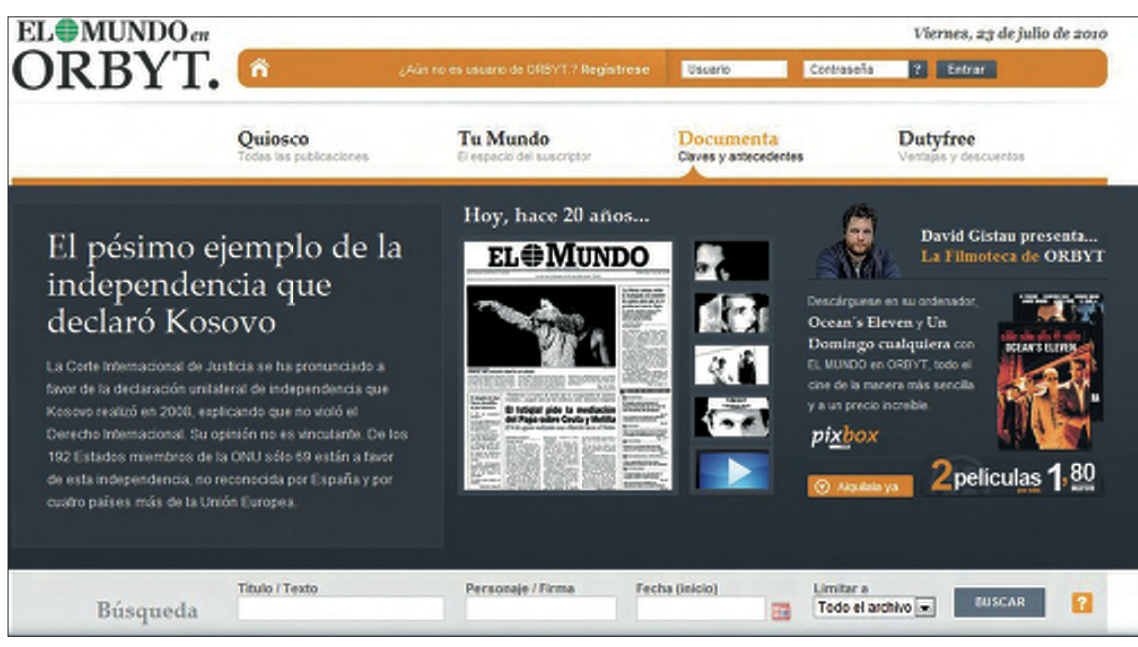

Orbyt, de El mundo, http://elmundo.orbyt.es/ vo sobre los resultados económicos fue notable, registrando unas pérdidas de 19,3 millones de euros entre 2003 y 2005 (Cea-Esteruelas, 2009). En 2006, con el regreso a la gratuidad, volvieron los números positivos.

La apuesta de Elpaís.com puso de manifiesto la solidez de la cultura de la gratuidad en el consumo de información online en España. La implantación del sistema de pago en este diario propició un importante trasvase de audiencia hacia medios que ofrecían noticias de libre acceso en la Red (Salaverría, 2007) y de ello se benefició a su principal competidor, Elmundo. es, que capitalizó al público reacio a pagar, asentado su liderazgo en internet. No sólo perdió capacidad de influencia respecto de la audiencia sino también en relación con otros medios. El fracaso de esta estrategia llevó a Elpaís.com a volver a un modelo predominantemente gratuito en junio de 2005, sólo dos años y medio después de instaurar el pago total. Esta experiencia demostró las complicaciones para el establecimiento de sistemas basados en el cobro íntegro por los contenidos informativos y la dificultad de planear este tipo cambios como tránsitos a corto plazo (Rojo-Villada, 2008).

\section{"La publicidad es insuficiente para garantizar los ingresos necesarios a la industria de la prensa en internet"}

Por su parte, Elmundo.es, pese a protagonizar los primeros intentos por introducir sistemas mixtos de pago en 2001 y 2002 (Salaverría, 2007), se ha caracterizado por un fuerte predominio de los contenidos gratuitos. Inicialmente permitía el libre acceso a las noticias elaboradas por y para la edición digital, mientras que restringía a los subscriptores las informaciones

procedentes del diario impreso y la hemeroteca. Este sistema le ha permitido liderar el ranking de la prensa online con un promedio diario de 376.284 usuarios únicos y casi 1,9 millones de visitas en 2008 (AEDE, 2009).

No obstante, la creciente caída de la venta de ejemplares en quioscos, que en el caso de la edición impresa de El mundo fue del $14 \%$ en el último año, ha abierto la puerta a una redefinición de su estrategia en internet. Así, en marzo de 2010 este diario apostó por la implantación de un modelo de negocio freemium denominado Orbyt. Se trata de una fórmula híbrida que combina la gratuidad, para noticias de última hora, con el pago por contenidos de valor añadido. Estos últimos están compuestos por los pdfs de las 20 ediciones del diario impreso y otros suplementos, vídeos y material multimedia, archivo y hemeroteca, la posibilidad de contactar directamente con la redacción y un club de ventas con descuentos. El acceso a este paquete se basa en una suscripción individual de 15 euros mensuales. Cuatro meses después de su lanzamiento, Orbyt ha acumulado 11.700 abonados, una cifra que supone unos ingresos de 175.500 euros mensuales y una previsión de 2,1 millones de euros anuales.

El objetivo de Elmundo.es con la aplicación de este modelo es maximizar el rendimiento de su actividad informativa diversificando los métodos de comercialización de su producto. Algo que sintoniza con la distribución multiplataforma de sus contenidos informativos que circulan no sólo por internet sino también a través de dispositivos móviles, como los lectores de libros electrónicos -iPad-o los teléfonos móviles. Esta última estrategia también ha sido adoptada por Elpaís.com y un 66\% de los medios españoles (Cabrera, 2010).

Pese a que es pronto para evaluar su efectividad, el sistema freemium permite a priori introducir una nueva vía de ingresos por la venta de contenidos, aunque de carácter complementario dada su modesta magni- 
tud. Pero sobre todo evita el riesgo de sufrir un retroceso del tráfico de usuarios que visitan el sitio web y el consiguiente descenso de la inversión publicitaria. La confianza en Orbyt ha provocado su extensión a Expansión.es, el diario económico perteneciente a Unidad Editorial, la empresa propietaria de Elmundo.es.

Las dificultades para consolidar un modelo de negocio viable en internet también afectan a los medios íntegramente online, que no tienen versión en papel. Entre éstos sobresalen los casos de Soitu y Factual. El primero fue impulsado en febrero de 2007 por Gumersindo Lafuente, quién fuera máximo responsable de Elmundo.es. El proyecto contó con el apoyo del banco $B B V A$, quién se convirtió en el socio de referencia acumulando un $49 \%$ de las acciones, frente al $51 \%$ que quedó en manos de los periodistas fundadores. La entidad financiera aportó 3,5 millones de euros al capital de este diario digital durante su existencia.

Soitu apostó por una fórmula innovadora de periodismo en internet, potenciando la información personalizada y la participación activa de los lectores. Su modelo de negocio se basó en la gratuidad y la obtención de ingresos vía publicidad. En septiembre de 2009 el medio contaba con un promedio diario de 42.000 usuarios únicos y 1,08 millones de visitas, según OJD. Su plantilla llegó a contar con 38 personas que luego quedaron reducidas a 23. La experiencia acabó a finales de octubre de 2009, después de 22 meses de actividad, tras el derrumbe de sus ingresos debido a la fuerte reducción del mercado publicitario provocada por la crisis económica.

Factual apareció en noviembre de 2009 con una clara apuesta por un modelo de negocio basado en el pago íntegro de todas sus informaciones. El sistema consistía en el cobro de 50 euros anuales por el acceso ilimitado a sus contenidos. El único dato disponible cifró en 700 personas el número de subscriptores, lo que arrojaba unos ingresos anuales de 35.000 euros.

\section{"Las experiencias de Soitu y Factual demuestran lo difícil que es consolidar una nueva marca de prensa digital”}

El proyecto fue una iniciativa del periodista Arcadi Espada, quién recibió el apoyo del grupo Quat que aportó un presupuesto inicial de 250.000 euros. El equipo inicial del periódico estaba formado por 20 personas. La trayectoria de Factual estuvo marcada no sólo por la escasez de audiencia sino también por la inestabilidad interna. Debido a desavenencias con la empresa propietaria y a la política de recortes presupuestarios, que redujeron la plantilla a 8 miembros, Espada sólo permaneció dos meses como director. Su salida desencadenó además un cambio de modelo de negocio, que se desplazó hacia la gratuidad. En su corto recorrido cuatro personas desempeñaron la máxima responsabilidad en este medio y tras nueve meses, en julio de 2010, Factual cerraba sus puertas. Tanto este caso como el de Soitu ponen de manifiesto los problemas para consolidar una nueva marca informativa en la prensa digital y reafirman las complicaciones para encontrar un modelo de negocio viable y rentable económicamente en la Red, bien sea de pago, que encuentra mayores resistencias, bien sea gratuito, con la fuerte dependencia publicitaria que entraña.

\section{Conclusiones}

La búsqueda de nuevos modelos de negocio se está convirtiendo en un objetivo clave de las empresas periodísticas de cara a garantizar la supervivencia de los diarios en el escenario de la convergencia digital. Sin embargo el establecimiento de nuevas fórmulas se topa con numerosos obstáculos que impiden que el aumento del consumo de información en internet se traduzca en un incremento de los ingresos económicos para los periódicos.

\section{"Internet permite la configuración de diversos modelos de negocio para la prensa: el gratuito, el de pago total, el metered model, el freemium y el basado en donaciones"}

Los factores que plantean estas trabas son, por un lado, el predominio de la cultura de la gratuidad en el acceso a las noticias online, enmarcada en un cambio de los hábitos de consumo del público, y el aumento de la competencia y, por ende, de los sitios web donde obtener información de actualidad. En el trasfondo la convergencia está impulsando un cambio en profundidad de la concepción de la información periodística que pasa de entenderse como un producto para configurarse como un servicio. Conviene no olvidar, además, la situación de crisis que vive el sector, más preocupado en reducir costes que en potenciar la innovación, y el declive de los anuncios en los diarios impresos unido al lento crecimiento de la inversión publicitaria en la Red.

La publicidad genera unos ingresos insuficientes para asegurar la viabilidad de la prensa en internet. 
Este hecho está orientando las estrategias de las empresas periodísticas hacia la implantación de sistemas de pago por el acceso a los contenidos informativos. En la actual coyuntura, donde predomina el ensayo-error, parece que las fórmulas mixtas, como el freemium, que combinan cobro y gratuidad, se alzan como la mejor solución. Mientras asistimos a su afianzamiento o a su fracaso, la ausencia de nuevos modelos de negocio solventes no sólo pone en aprietos el futuro de la prensa en internet sino que complica la consolidación de nuevos proyectos periodísticos en la Red.

\section{Bibliografía}

AEDE (Asociación de Editores de Diarios de España). Libro blanco de la prensa diaria 2010. Madrid: AEDE, 2009.

Anderson, Chris. Free: The future of a radical price. New York: Hyperion, 2009, ISBN 978-1-4013-2290-8.

Albarran, Alan B. The media economy. New York: Routledge, 2010, ISBN 978-0-415-99046-2.

Boczkowski, Pablo. Digitalizar las noticias. Innovación en los diarios online. Buenos Aires: Manantial, 2006, ISBN 978-987-500-094-0.

Cabrera, María-Ángeles. “Introducción”. En: Cabrera, María Ángeles (coord.). Evolución tecnológica y cibermedios. Zamora: Comunicación Social, 2010, pp. 9-20. ISBN 9788492860418.

Cea-Esteruelas, María-Nereida. "Modelo de negocio de la empresa periodística en internet: el caso de Prisa". Revista latina de comunicación social, 2009, n. 64, pp. 938-950, http://www.revistalatinacs.org/09/art/872_ UMA/73_99_Nereida_Cea_Esteruelas.html

Deuze, Mark. "Media industries, work and life". European journal of communication, 2009, v. 24, n. 4, pp. 467-480.

Díaz-Noci, Javier. "2018: ¿Diarios en dispositivos móviles? Libro electrónico, tinta electrónica y convergencia de la prensa impresa y digital". El profesional de la información, 2009, mayo-junio, v. 18, n. 3, pp. 301-307.

Dornelles, Beatriz. "O futuro do jornal”. Famecos, 2009, dic., n. 40, pp 63-67.

García-Avilés, José-Alberto. "La comunicación ante la convergencia digital: algunas fortalezas y debilidades". Signo y pensamiento, 2009, n. 54, pp. 102-113.

Infoadex. Estudio Infoadex de la inversión publicitaria en España 2010. Madrid: Infoadex, 2010.

Jenkins, Henry. Convergence culture. La cultura de la convergencia de los medios de comunicación. Barcelona: Paidós, 2008, ISBN 978-84-4932153-5.

Larrañaga, Julio. "La crisis del modelo económico de la industria de los periódicos". Estudios sobre el mensaje periodístico, 2009, n. 15, pp. 61-80.

Lawson-Borders, Gracie. Media organizations and convergence. Case studies of media convergence pioneers. New Jersey: Lawrence Erlbaum, 2006, ISBN 0-8058-5032-5.

López, Xosé; Pereira, Xosé. "La prensa ante el reto online. Entre las limitaciones del modelo tradicional y las incógnitas de su estrategia digital". En: Bustamante, Enrique et al. Alternativas en los medios de comunicación digitales. Barcelona: Gedisa, 2008, pp. 177-244, ISBN 978-84-9784-333-1.

Marcos-Recio, Juan-Carlos; Edo, Concha; Flores, Jesús; García-Alonso, Pedro; Parra, David. "Estrategias y perspectivas documentales en la información digital". Ámbitos: Revista intl de comunicación, 2008, n. 17, pp. 9-23.

Martínez-Molina, Matías. "La crisis de los grandes periódicos". Infoamérica, 2010, n. 2, pp. 139-148.

Mateo, Rosario; Bergés, Laura; Garnatxe, Anna. "Crisis ¿qué crisis? Los medios de comunicación: empresas y periodismo en tiempos de crisis". En: Campos-Freire, Francisco (coord.). El cambio mediático. Zamora: Comunicación Social, 2010, pp. 75-106, ISBN 978-84-92860-25-8.

Meyer, Philip. "El periódico élite del futuro". En: Espada, Arcadi; Hernández-Busto, Ernesto (eds.). El fin de los periódicos. Barcelona: Duomo, 2009, pp. 33-40, ISBN 978-84-92723-11-9.

Micó-Sanz, Josep-Lluís; González-Molina, Sonia. "Convergencia en la comunicación empresarial en las oficinas de prensa de tráfico y seguridad vial". El profesional de la información, 2010, marzo-abril, v. 19, n. 2, pp. 133-139.

Micó-Sanz, Josep-Lluís; Masip, Pere; Barbosa, Suzana. "Models of business convergence in the information industry: A mapping of cases in Brazil and Spain”. Brazilian journalism research, 2009, v. 5, n. 1, pp. 123-140.

Miguel-de-Bustos, Juan-Carlos; Casado-del-Río, Miguel-Ángel. "Sobre pago y gratuidad. Momentos de crisis en la industria periodística”. Telos. Cuadernos de comunicación e innovación, 2010, n. 83, pp. 24-35.

Mitchelstein, Eugenia; Boczkowski, Pablo. "Between tradition and change: A review of recent research on online news production". Journalism, 2009, v. 10, n. 5, pp. 562-586.

Mosco, Vincent. The political economy of communication. London: Sage, 2009, $2^{\text {a }}$ ed., ISBN 978-1-4129-4701-5.

OCDE (Organización para la Cooperación y el Desarrollo Económico). The evolution of news and the internet. Informe DSTI/ICCP/IE(2009)14/Final, 2010

Palacios, Marcos; Díaz-Noci, Javier. Ciberperiodismo: métodos de investigación. Una aproximación multidisciplinar en perspectiva comparada. Bilbao: Universidad del País Vasco, 2009, ISBN 978-84-9860-191-6.

PEJ (Project for Excellence in Journalism). State of the news media 2010 The Pew Research Center, 2010, http://www.stateofthemedia.org/2010/index.php

PriceWaterhouseCoopers. Global Entertainment and Media Outlook: 20092013. Madrid: PWC, 2009a.

http://www.pwc.com/es/gemo

PriceWaterhouseCoopers. Moving into multiple business models. Outlook for newspaper publishing in the digital age, 2009b.

http://www.pwc.com/e\&m

Rodríguez-Martínez, Ruth; Codina, Lluís; Pedraza-Jiménez, Rafael. "Cibermedios y web 2.0: modelo de análisis y resultados de aplicación". El profesional de la información, 2010, enero-febrero, v. 19, n. 1, pp. 35-44.

Rojo-Villada, Pedro-Antonio. Modelos de negocio y consumo de prensa en el contexto digital. Murcia: Universidad de Murcia, Servicio de Publicaciones, 2008, ISBN 978-84-8371-744-8.

Salaverría, Ramón. "Ciberperiodismo: diez años de prensa digital en España”. En: Fernández, Juan-José (coord.). Prensa especializada actual. Doce calas. Madrid: McGraw-Hill, 2007, pp. 355-383.

Salaverría, Ramón; Negredo, Samuel. Periodismo integrado. Barcelona: Editorial Sol90, 2008, ISBN 978-84-9820-954-9.

Salaverría, Ramón; García-Avilés, José-Alberto. "La convergencia tecnológica en los medios de comunicación: retos para el periodismo". Trípodos, 2008, n. 23, pp. 31-47.

Sánchez-Tabernero, Alfonso. "La prensa en Europa: claves de un sector estancado". Telos. Cuadernos de comunicación e innovación, 2008, n. 75 , pp. 104-106.

Sonnac, Nathalie. "L'économie de la presse: vers un nouveau modèle d'affaires". Les cahiers du journalisme, 2009, n. 20, pp. 22-43.

WAN (World Association of Newspapers). "The paid vs. free content debate". Shaping the future of the newspaper, Strategy report, 2010, March, v. 9 , n. 2, pp. 1-38.

Andreu Casero-Ripollés. Departamento de Ciencias de la Comunicación, Universitat Jaume I de Castelló, Castelló de la Plana.

casero@com.uji.es 